\title{
Blur: Solar Multi-Conjugate Adaptive Optics Simulations
}

\author{
Jose Marino and Dirk Schmidt \\ National Solar Observatory, Boulder CO, USA
}

\begin{abstract}
We present a study to evaluate and predict the performance of a solar multi-conjugate adaptive optics (MCAO) system using computer simulations. Blur is an end-to-end solar AO/MCAO simulation package capable of fast and accurate MCAO simulations. We use Blur together with KAOS, a solar AO/MCAO controller, to simulate the MCAO system Clear, a pathfinder solar MCAO system at the $1.6 \mathrm{~m}$ Goode Solar Telescope (GST) at the Big Bear Solar Observatory (BBSO), and evaluate its performance under different conditions.
\end{abstract}

\section{INTRODUCTION}

Multi-conjugate adaptive optics (MCAO) promises to deliver adaptive optics (AO) correction over a wider field-of-view (FOV). This technique is particularly important for solar observations where high resolution images of the Sun, usually extending several tens of arcseconds, are commonly required. Single conjugate, or classical, solar AO systems are routinely used in current solar observatories. Seeing conditions during the day are much more severe than during the night due to the heating of the ground by the Sun. Moreover, solar telescopes operate in the visible range of the spectrum where the effects of atmospheric turbulence are much more pronounced. Solar AO systems have enabled solar ground based observations to achieve diffraction limited high resolution observations of the Sun.

The correction provided by a solar AO system is usually limited to a small circular area around the lock point of the $\mathrm{AO}$ system with a typical size of $10^{\prime \prime}$, the isoplanatic patch. Beyond this area, the correction provided by the AO system degrades rapidly producing a solar image with a sharp high resolution center surrounded by progressively distorted edges. This occurs because the AO system measures the incoming wavefront distortions only along a single field direction the lock point of the $\mathrm{AO}$ system- but applies the required correction using a deformable mirror (DM) conjugated to the entrance pupil of the telescope, and thus applying it to all field directions equally. Atmospheric turbulence occurs over the full volume of the atmosphere and not just close to the ground where the DM is conjugated. Thus, the applied wavefront correction -valid at the lock point- is not valid for other field directions which travel through different volumes of the atmosphere. The image quality of the $\mathrm{AO}$ corrected image is good at the lock point but it progressively degrades as we move away from it. MCAO systems rely on several DMs conjugated to different heights in the atmosphere to achieve a more volumetric correction of the atmospheric distortions. MCAO also requires wavefront measurements along different field directions to prove a wider volume of the atmosphere and tomographically reconstruct the distortions at the conjugated heights of the extra DMs.

Solar AO is a well established technology used routinely at many night time observatories and practically all solar observatories. ${ }^{1}$ Solar MCAO is a relatively new technology still in the 
development stage $^{2}$ with several experimental solar MCAO systems currently being developed, such as Clear. However, the next generation of solar telescopes represent an extreme improvement over current facilities. State of the art current solar telescopes have aperture sizes in the range of $1.5 \mathrm{~m}$, while the next generation of solar telescopes like the DKIST and EST have aperture sizes of $4 \mathrm{~m}$. The AO systems required for this next generation AO systems are major technological upgrades with thousands of degrees of freedom. The AO system for the Daniel K. Inouye Solar Telescope (DKIST, formerly known as ATST) currently under construction will have a DM with 1,600 actuators. These large solar AO and MCAO systems will benefit from simulation tools that can accurate simulate their performance and aid in their design and operation.

\section{BLUR: SOLAR ADAPTIVE OPTICS SIMULATION PACKAGE}

Blur is an end-to-end solar AO/MCAO simulation package developed in house at the National Solar Observatory (NSO) that takes advantage of current multi-core computer systems to parallelize computation. Blur aims to provide fast and accurate solar AO and MCAO simulations. It is designed with flexibility in mind to allow the simulation of any solar AO system, from conventional AO to MCAO systems with several mirrors and wavefront sensors (WFS). The simulation package has a modular design that allows the operation of the system to be performed by separate components that take care of the physical simulation and the AO control, respectively. The control of the AO simulated system is currently handled by KAOS, a state of the art solar AO control software developed by the Kiepenheuer-Institut fuer Sonnenphysik (KIS). KAOS is a robust and stable control system which is routinely used in existing solar telescopes to control their AO systems, such as the $1.5 \mathrm{~m}$ GREGOR telescope in Tenerife, Spain. KAOS provides a tried and tested controller that allows us to concentrate on the physical simulation part, provided by Blur, and improves the accuracy of the AO simulations. Additionally, Blur can be used as a flexible testbed for development of KAOS without having to rely on a real AO system.

Our solar AO simulation package, Blur, is capable of extremely accurate solar AO simulations. This is achieved by performing a full simulation of the imaging process that takes place in a solar Shack-Hartmann wavefront sensor (SHWFS). Each of the sub-aperture images is computed by simulating the actual imaging process that takes place in a real SHWFS lenslet, including field dependent imaging. Traditionally, solar AO simulation packages do not perform these very computationally intensive calculations. They rely instead on approximations that attempt to emulate the wavefront sensing process in a more efficient way. However, such a trade-off hurts the accuracy of the simulations by failing to include some very important side effects of the imaging and crosscorrelation process that take place in a real solar AO system, such as field-of-view distortions and varying contrast. In Blur, the effects of WFS noise are included at the imaging level and allowed to propagate to the SHWFS measurements as they would do in a real SHWFS system. Shot noise and read noise with random characteristics are applied to every SHWFS frame. By taking advantage of current multi-core computer systems we can run these computations in parallel and achieve good simulation performance without sacrificing the accuracy of the simulations.

Blur is written in C++11. It uses OpenMP (Open Multi-Processing) to produce multi-threaded code that can take advantage of current multi-core computer systems to produce efficient parallel computations. OpenMP ${ }^{3}$ provides an easy to use and powerful programming interface that enables shared memory multiprocessing programming in C++. Blur's two main external dependencies are the armadillo library, ${ }^{4}$ a linear algebra library, and the FFTW library, ${ }^{5}$ a fast Fourier transform 
library. Blur can also be controlled from a Python interface, which allows for easy scripting capabilities and automation of complex and repetitive AO simulation tasks.

\section{CLEAR: PATHFINDER MCAO SYSTEM}

Clear is a pathfinder solar MCAO system ${ }^{2}$ under development at the Goode Solar Telescope (GST), formerly known as the New Solar Telescope (NST), at the Big Bear Solar Observatory (BBSO) in Big Bear, CA. Clear is a collaboration effort between NSO, BBSO (operated by the New Jersey Institute of Technology), and KIS. The aim of the system is to demonstrate solar MCAO using the $1.6 \mathrm{~m}$ aperture GST and to serve as an experimental platform to explore a future solar MCAO system for the DKIST.

Clear is designed to have a flexible configuration using a dedicated tip-tilt mirror, three identical DMs - each with 357 actuators- and a wide field SHWFS. One of the DMs is always conjugated to the entrance pupil of the telescope while the other two DMs are conjugated to heights between 2 and $9 \mathrm{~km}$ above the ground. The high altitude DMs are arranged in a double-z configuration ${ }^{6}$ on the optical table, which allows the DMs to be positioned at different conjugated heights inside the previously mentioned range. The wide field SHWFS can be configured to have a range of FOVs and number of subapertures. A square grid lenslet array forms an array of sub-aperture images of the solar surface with a large FOV. Several sub-regions inside each sub-aperture image are extracted to be cross-correlated independently in order to provide WFS measurements along different field directions. In the particular SHWFS configuration simulated in this study, the SHWFS has 208 subaps located in a $16 \times 16$ grid with $3 \times 3$ sub-regions inside each sub-aperture image spaced by $11.4^{\prime \prime}$, with the center sub-region located on-axis. The FOV imaged by the SHWFS and the number of sub-apertures across are determined by the cameras currently available. Ideally, a large number of sub-apertures and a large FOV are both desirable to obtain high resolution WFS measurements over a large FOV. However, the number of pixels available in the camera places an upper limit that forces a balance between the SHWFS parameters. A large FOV, i.e., more pixels per sub-aperture can be achieved at the cost of less sub-apertures across the pupil. Conversely, more sub-apertures can be achieved when the number of pixels per sub-aperture is reduced, i.e., smaller FOV size in each sub-aperture.

The physical sizes of the DMs when projected on their conjugated heights are not optimized to the FOV area sensed by the SHWFS. This is due to the physical constrains of the optical laboratory at the GST and to facilitate the flexibility required to easily move the DMs to different heights. In most situations, the projected size of the DMs is larger than the area sensed by the SHWFS leading to unseen actuators on the edges of the DMs. These unseen actuators can be disabled depending on the chosen configuration and the actual projected sizes. Additionally, the size of the pupil is reduced to $1.42 \mathrm{~m}$ right after the MCAO system. This is to minimize the intensity fluctuations caused by pupil distortions introduced by the high altitude DMs. ${ }^{6}$

\section{SIMULATION OF CLEAR}

The AO simulation package, Blur, was set up to simulate a solar MCAO system with the exact configuration as Clear. The $3 \mathrm{DMs}$ were configured conjugated to $0 \mathrm{~km}, 3 \mathrm{~km}$, and $8 \mathrm{~km}$. The physical size of the DMs conjugated to the layers were $1.52 \mathrm{~m}, 2.40 \mathrm{~m}$, and $3.49 \mathrm{~m}$, repectively. The SHWFS was configured with a square grid $(16 \times 16)$ lenslet array, each lenslet producing an image of the Sun with $37.2^{\prime \prime}$. The number of useful sub-apertures that fall inside the telescope 

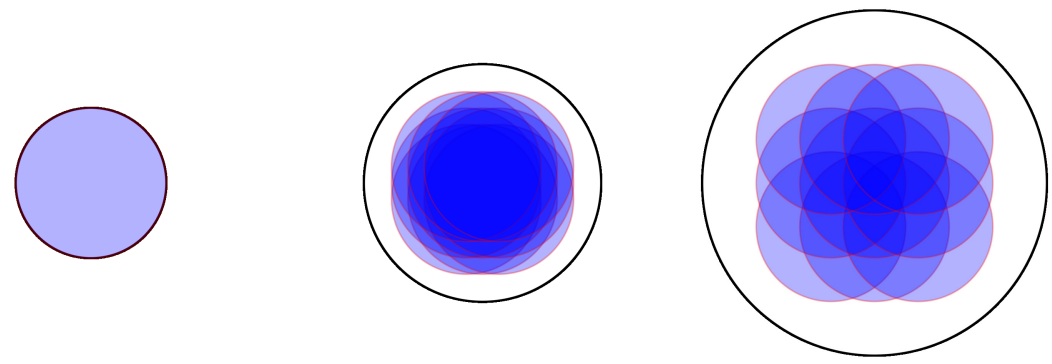

Figure 1: Telescope pupil projections for all sensing directions overlayed at the conjugated heights of each DM: at $0 \mathrm{~km}$ (left), $3 \mathrm{~km}$ (center), and $8 \mathrm{~km}$ (right). The black solid circles indicate the physical size of each DM at its conjugated height.
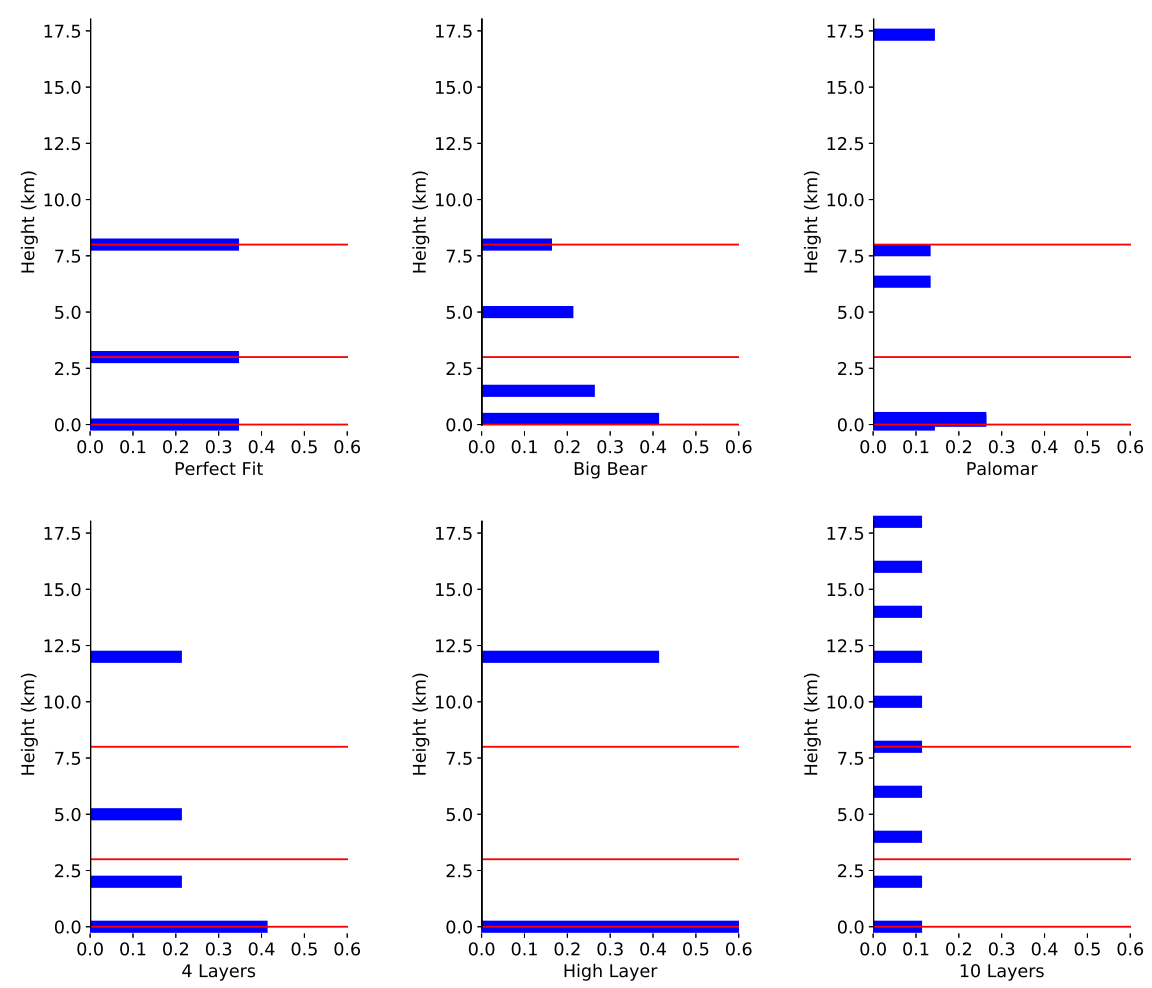

Figure 2: Atmospheric models used in the simulations. Blue bars indicate the location and strength (bar length) of the turbulent layers. Red lines indicate the conjugated heights of the DMs.

pupil is 208. The SHWFS camera was configured with a pixel scale of $0.62^{\prime \prime} /$ pixel so that each sup-aperture image uses $60 \times 60$ pixels to produce the desired $37.2^{\prime \prime}$ FOV sub-aperture images. Nine separate regions placed in a $3 \times 3$ grid located $11.4^{\prime \prime}$ apart are extracted from each sub-aperture image to produce WFS measurements along 9 different directions. Thus, the SHWFS produces 208 wavefront sensor measurements along 9 directions located on a square grid, producing a total of $208 \times 9=1872$ wavefront measurements. 
The projected sizes of the DMs at their conjugated layers were chosen to match the actual design of Clear. The DM sizes, show in Fig. 1 are not fully optimized to the metapupils due to space and complexity limitations in the optical design. Thus, the sizes of the DMs are larger than the sizes of the metapupils at each conjugated height leading to unseen actuators around the outside ring of the high altitude DMs that were not actively driven by the control system. In the simulation setup, only the seen actuators in each DM are considered and simulated. The number of actuators used in each DM is $241(17 \times 17), 177(15 \times 15)$, and $137(13 \times 13)$ for the pupil, $3 \mathrm{~km}$, and $8 \mathrm{~km} \mathrm{DMs}$, respectively. The final pupil size of the MCAO system was stopped down to $1.42 \mathrm{~m}$ as in the actual design of Clear. The stopping down of the pupil in the real system is done to minimize intensity fluctuations caused by pupil distortions that are introduced by the high altitude DMs. It should be noted that the simulation code is not capable at this time to reproduce these intensity fluctuations, however, the specifications were kept as close as possible to the actual design specifications of Clear.

The AO controller software KAOS was configured to perform 3 separate control schemes: classical AO, ground-layer AO (GLAO), and MCAO. Classical AO uses only the WFS measurements along the on-axis direction, i.e., the lock point, to control only the pupil DM, conjugated to the entrance pupil of the telescope. The GLAO scheme uses the information provided by all the WFS sensing directions to estimate the optimal wavefront distortions to be applied to the pupil DM. GLAO correction aims to maximize the size of the AO corrected FOV at the expense of a lower correction over all. The MCAO control scheme uses the information from all the WFS sensing directions to drive all the $3 \mathrm{DMs}$ conjugated to various heights above the telescope. The control performed by KAOS is a modal control using Karhunen-Loève (KL) modes, ${ }^{7}$ correcting $147 \mathrm{KL}$ modes, $50 \mathrm{KL}$ modes, and $40 \mathrm{KL}$ modes on the pupil, $3 \mathrm{~km}$, and $8 \mathrm{~km}$ DMs, respectively.

Each simulation run starts with a random atmospheric realization and runs the MCAO control loop in each of the 3 control schemes sequentially. After a few iterations a performance measurement is obtained, for each of the 3 control schemes. The process is repeated for 200 different random realizations of the atmospheric model to obtain a good performance average. The simulation data was generated taking advantage of the flexible Python interface to Blur, which allows the easy repetition of each simulation setup for a series of random atmospheric model realizations.

\subsection{Atmospheric models}

Performance estimates were computed for a set of different atmospheric models shown in Fig. 2. Each atmosphere model in the simulations was implemented by a set of discrete random Kolmogorov phase screens. The phase screens were computed by the Fourier transform method $^{8}$ and stored to disk. Low order harmonics were included to improve the statistical properties of the phase screens in the low spatial frequency ranges.

The atmospheric models tested include a "Perfect Fit" model where the atmospheric layers are located exactly at the same heights where the DMs are conjugated. This should provide a best case scenario for the MCAO controller. The "Big Bear" atmospheric model is based on the measurements produced by an atmospheric profile experiment ${ }^{9}$ at the site of the GST, where Clear is being developed. Similarly, the "Palomar" was measured at the Palomar site in southern California, ${ }^{10}$ which is geographically very close to the site of GST. The other 3 atmospheric models were chosen to explore the performance under unfavorable or very extreme atmospheric condition, where none of the turbulent layers fall at a DM conjugated height. All atmospheric models have a global Fried paramater of $\mathrm{r}_{0}=15 \mathrm{~cm}$. 

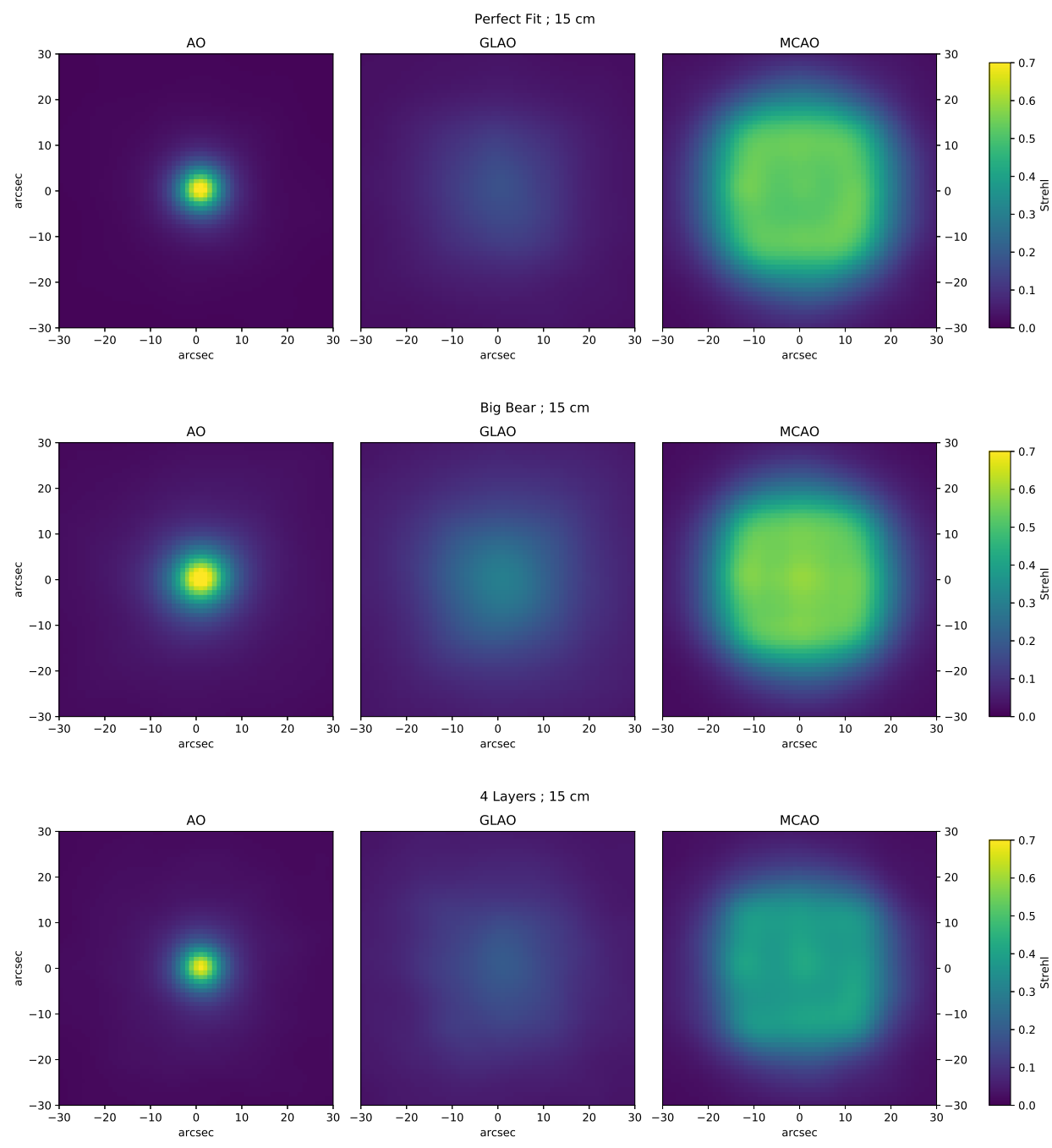

Figure 3: Average Strehl ratio maps over a $60^{\prime \prime}$ square field-of-view for 3 atmospheric configurations. The left, middle, and right columns show Strehl ratio maps with the system running in classical AO mode, GLAO mode, and MCAO mode, respectively.

\section{RESULTS}

The performance of the simulated MCAO system was quantified as the average residual rms wavefront error measured along a grid of $61 \times 61$ field directions covering a FOV of $60 \times 60^{\prime \prime}$, i.e., data samples spaced by $1^{\prime \prime}$. The Strehl ratio can be estimated from the residual rms wavefront using the Marechal approximation. ${ }^{11}$ Fig. 3 shows the results obtained for 3 of the atmospheric models described in Fig. 2. Each row in Fig. 3 shows the Strehl ratio across the FOV for each of the 3 control schemes implemented in KAOS: AO, GLAO, and MCAO. The AO Strehl maps (left panes) illustrate the typical performance of a solar AO system, the Strehl ratio is very high along the on-axis 
direction -the direction measured by the SHWFS- and it sharply drops for field directions away from it. The 3 panels in Fig. 3 also illustrate the effect on the size of the isoplanatic patch of the distribution of high altitude turbulence. Stronger high altitude turbulence will produce a smaller isoplanatic patch. The GLAO Strehl maps (middle panes) show a much lower overall Strehl ratio but with a wider distribution. Ideally, a GLAO system would produce a flat Strehl ratio map where every field direction has the same level of correction, i.e., the same Strehl ratio. However, the relatively small FOV of the SHWFS limits the effectiveness of the GLAO correction and reduces the field size of the corrected area. The MCAO Strehl maps (right panes) show a high Strehl ratio over a much larger FOV with a size determined by the FOV sensed by the SHWFS.

Fig. 4 shows azimuthal averages of the Strehl maps. Each of the 5 plots compares the achieved performance, i.e., Strehl ratio, under all the atmospheric configurations tested against the performance achieved under an ideal configuration where the DMs are located exactly at the same heights as the turbulent layers. The variation of the size of the isoplanatic patch with atmospheric configuration is much more clearly visible in the azimuthal average plots. The strength of high altitude turbulence is lower in the "Big Bear" atmospheric model than in the "Perfect Fit" model, which produces a larger isoplanatic patch in the AO Strehl ratio, as shown in the top-left pane of Fig. 4. Conversely, the strength of high turbulence is larger in the "High Layer" and "10 Layers" configurations, producing smaller AO corrected isoplanatic patches. The GLAO performance also shows a dependence on high altitude turbulence strength, producing flatter Strehl profiles for stronger high turbulence. The performance of the MCAO system is also heavily dependent on the altitude of the turbulence and its strength. The MCAO system performs best when the turbulent layers are close to the conjugated heights of the DMs and its performance degrades fast for atmospheric profiles with strong high altitude turbulence located far from the conjugated DM heights.

\section{CONCLUSIONS}

Solar AO and MCAO simulation provides a powerful tool to study the expected performance of the next generation of solar $\mathrm{AO}$ and MCAO systems. It is a valuable tool to help understand the design requirements of solar MCAO systems. The simulations show how the location and strength of the high altitude turbulence has a strong effect on the performance achieved by the solar MCAO system. It is very important to have accurate and up-to-date profile measurements of the atmospheric turbulence distribution over the telescope in order to optimize the design of the MCAO system. Currently, solar observatories do not have the capabilities to routinely obtain

atmospheric profile measurements. These will become vital once solar MCAO systems become a mature technology that can be deployed to solar observatories around the world.

\section{ACKNOWLEDGMENTS}

This work was funded by the Association of Universities for Research in Astronomy, the National Solar Observatory and the National Science Foundation.

\section{REFERENCES}

[1] Rimmele, T. R. and Marino, J., "Solar Adaptive Optics," Living Reviews in Solar Physics 8 (June 2011). 

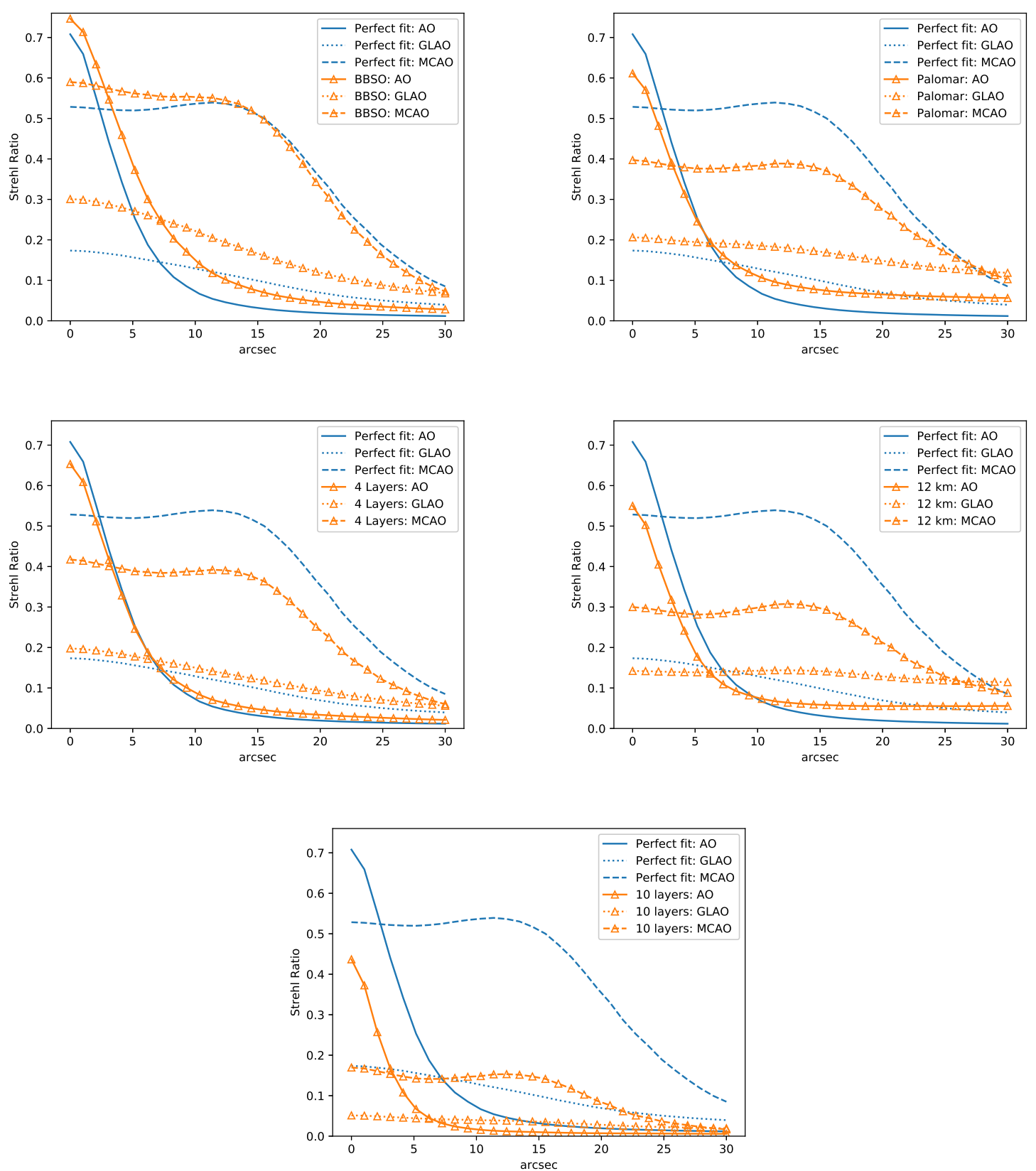

Figure 4: Azimuthal average Strehl ratios. Each plot compares the Strehl ratios from one of the atmospheric models against the "Perfect Fit" atmospheric model. 
[2] Schmidt, D., Gorceix, N., Goode, P. R., Marino, J., Rimmele, T., Berkefeld, T., Wöger, F., Zhang, X., Rigaut, F., and von der Lühe, O., "Clear widens the field for observations of the Sun with multi-conjugate adaptive optics," A\&A 597 (Jan. 2017).

[3] OpenMP Architecture Review Board, "OpenMP application program interface version 4.5," (May 2015).

[4] Sanderson, C., "Armadillo: An open source c++ linear algebra library for fast prototyping and computationally intensive experiments," tech. rep., NICTA (2010).

[5] Frigo, M. and Johnson, S. G., "The design and implementation of FFTW3," Proc. IEEE 93(2), 216-231 (2005).

[6] Schmidt, D., Gorceix, N., Marino, J., Berkefeld, T., Rimmele, T., Zhang, X., Wöger, F., and Goode, P., "Progress in multi-conjugate adaptive optics at Big Bear Solar Observatory," Proc. SPIE 9909 (July 2016).

[7] Dai, G.-M., "Modal compensation of atmospheric turbulence with the use of zernike polynomials and karhunen-loève functions," JOSA A 12, 2182-2193 (1995).

[8] Johansson, E. M. and Gavel, D. T., "Simulation of stellar speckle imaging," Proc. SPIE 2200, 372-383 (June 1994).

[9] Kellerer, A., Gorceix, N., Marino, J., Cao, W., and Goode, P. R., "Profiles of the daytime atmospheric turbulence above Big Bear solar observatory," A 8 A 542 (June 2012).

[10] Wang, L., Schöck, M., and Chanan, G., "Atmospheric turbulence profiling with slodar using multiple adaptive optics wavefront sensors," App. Opt. 47, 1880-1892 (Apr. 2008).

[11] Hardy, J. W., [Adaptive Optics for Astronomical Telescopes], Oxford University Press (1998). 